\title{
Drug discovery, development and delivery in Alzheimer's disease
}

\author{
Davide Brambilla '
}

Received: 7 December 2017 / Accepted: 7 December 2017 / Published online: 29 December 2017

(C) Springer Science+Business Media, LLC, part of Springer Nature 2017

This Theme Issue of Pharmaceutical Research collects a series of original articles and literature reviews, providing a representative overview of the broad spectrum of research which is currently performed in the quest for an effective therapeutic option for Alzheimer's disease (AD), the most common cause of dementia, firstly described by Alois Alzheimer in $1906(1,2)$. Despite an extraordinary scientific effort during the last decades, AD remains one of the great challenges in medicine, with enormous societal and economic impact: it is estimated that forty million people are currently affected worldwide, with a total economic burden exceeding 500 billion $\$$ per year (3-6). Notably, as aging is a key risk factor, numbers are anticipated to drastically increase during the next decades, as a consequence of the overall increment in average life expectancy (3-5). According to The Alzheimer's Association, between 2000 and 2013, deaths resulting from stroke, heart disease, and prostate cancer decreased by $10-20 \%$, whereas deaths attributed to $\mathrm{AD}$ increased by $70 \%$. If this data reflect on one hand an increased awareness (proportion of death listing $\mathrm{AD}$ as the underlying cause), on the other, they highlight an overall pharmaceutical stagnation and the still unmet medical need, that likely originate from the complex and multifaceted nature of the disease (7). From a clinical perspective, while the initial symptom is a gradual worsening of the ability to store new information due to the damaging of memory-related neurons; later symptoms involve other cognitive functions, such as problem solving and communication skills, drastically affecting everyday life and eventually leading to death in a decadeslong process. Histopathologically, AD brains present two

Guest Editor: Davide Brambilla

Davide Brambilla

davide.brambilla@umontreal.ca

Faculté de Pharmacie, Université de Montréal, Montréal, Québec, Canada major hallmarks: the extracellular amyloid deposits, deriving their name from their visual similarities to starch (amylum), and intracellular tangles composed of hyperphosphorylated tau protein. In 1984, it was discovered that the amyloid plaques were mostly of peptidic nature (amyloid peptides) (8), setting the ground for the amyloid cascade hypothesis: the accumulation of toxic concentrations of amyloid peptides leads to initial neuronal impairment as well as the hyperphosphorylation and tangling of intracellular tau, further promoting neuronal damage, in a vicious degenerative cascade. Despite the fact that the hypothesis - supported by the observation that alterations of the amyloid peptide biogenesis cascade lead to $\mathrm{AD}$, while tau mutations result in clinically distinct pathologies (tauopathies) has gone through numerous implementations attempting to take into account accumulating new knowledge, thus far, a clear understanding of the disease aetiology and its progress pathway remains elusive (9). While the known genetic forms of $\mathrm{AD}$, including dominant mutations of the presenilins and the amyloid precursor protein, have been important in unraveling some mechanism behind the pathogenesis of this disease, they only account for $5 \%$ of cases, with the vast majority being of sporadic form. Concomitantly, the identification for risk factors has been the focus of large epidemiological studies, resulting in the determination of genetic (e.g., ApoE4 isoform), lifestyle habits (e.g., smoking, physical inactivity) and medical conditions (e.g., strokes, diabetes and hypertension) favoring the onset of $\mathrm{AD}$, providing hope for new therapeutic targets, preventive lifestyle guidelines and off-label drug utilization (10). Despite this, disease-changing treatments are severely lacking, with only symptomatic treatments, i.e. acetylcholine esterase inhibitors and an NMDA receptor antagonist, available for patients (6).

Several curative therapeutic strategies, targeting disparate aspects of the pathology, have been proposed and have shown promising results in preclinical models (11). Nevertheless, only a limited number (e.g. amyloid-targeted immunotherapy, secretases inhibitors and modulators) have gone through clinical testing, and all have failed to provide convincing 
therapeutic effects and to gain clinical approval $(12,13)$. Although this general failure might have multiple causes, including the limited predictive nature of the used preclinical models (14), consensus in the scientific community exists on the importance of reliable diagnostic tools for this disease, as symptoms can appear up to 20 years after the first biochemical changes. Currently, great effort and hope are thus placed on the identification of reliable biomarkers and standardized quantification methods, as well as on the design of selective and sensitive imaging probes and techniques able to identify $\mathrm{AD}$ at the earliest stage $(6,15)$. In general, it appears obvious that more fundamental research is required to better elucidate the biological mechanisms behind the disease and to identify suitable therapeutic targets, and allow for drug design and development to deliver effective therapeutic options.

This Theme Issue of Pharmaceutical Research collects three review and five original articles covering a large spectrum of topics relevant to the biomedical research on $\mathrm{AD}$. As stated above, literature consistently suggests an increased incidence of $\mathrm{AD}$ in diabetic patients. As a results of this correlation, Markowicz-Piasecka and colleagues provide an updated overview of this dualism and analyze the available data concerning the potential use of metformine, a first-line medication for the treatment of type 2 diabetes, as a possible treatment for $\mathrm{AD}$, while also depicting the relevant mechanisms. Protein aggregation plays a key role in the pathogenesis and progression of the disease. Paolo Arosio introduces and analyzes the literature pertinent to the complex biophysics governing amyloid aggregation, and discusses the currently assessed aggregation modifiers as a potential treatment. The review manuscript from Storck and Pietrzik outline the recent findings concerning the Low-Density Lipoprotein Receptor-Related Protein 1, a receptor frequently reported as having a key role in $\mathrm{AD}$. As accurately described by the authors, its involvement in the biogenesis of amyloid peptides as well as in their clearance from the brain make this receptor an essential player in the game and an interesting therapeutic target. The authors also discuss the available literature on its utilization as target for drug delivery applications. As recently reported, one important limitation preventing the identification of a well-defined aetiology mechanism and therapy, stem from the limitations of currently available $\mathrm{AD}$ animal models. Addressing this important aspect, Vellonen and colleagues investigated the expression of several transporters and molecules of the blood-brain barrier (BBB) tight-junctions in three different $\mathrm{AD}$ mice models and their comparison with healthy animals, as a crucial step to better characterize the available models. Delivery of therapeutics through the BBB is a great challenge for brain diseases including $\mathrm{AD}$, the work from Shah et al. reports the design of an intranasal micro-emulsion formulation as a platform for an efficient delivery across the BBB. The authors evaluated the performance of the delivery tool using rivastigmine as model drug. The work from Perrone and collaborators, investigated the ability of verapamil, a commonly used voltage-dependent calcium channels blocker, to prevent the phosphorylation of tau, a putative important event in $\mathrm{AD}$ aetiology. Finally, the articles from Ordonez et al. and Muench and colleagues, describe the investigation of dihydroceramide desaturase 1 inhibitors and tenilsetam, a radical scavenger and an anti-oxidant, as potential therapeutic molecules for $\mathrm{AD}$, as well as the dissection of the mechanistic behind their effect. To conclude, while we are aware that it does not represent an exhaustive illustration of currently implemented AD research, we are confident that this Issue gives an interesting and clear overview of the field, and wish you a good read.

ACKNOWLEDGMENTS AND DISCLOSURES. DB thanks Dr. V. Agostoni and S. Babity for proofreading.

\section{REFERENCES}

1. Barker WW, et al. Relative frequencies of Alzheimer disease, Lewy body, vascular and frontotemporal dementia, and hippocampal sclerosis in the State of Florida Brain Bank. Alzheimer Dis Assoc Disord. 2002;16:203-12.

2. Hippius H, Neundörfer G. The discovery of Alzheimer's disease. Dialogues Clin Neurosci. 2003;5:101-8.

3. Gaugler J, et al. 2016 Alzheimer's disease facts and figures. Alzheimers Dement. 2016;12:459-509.

4. Stefanacci RG. The costs of Alzheimer's disease and the value of effective therapies. Am J Manag Care. 201 1;17(Suppl 1):S356-62.

5. Maresova $\mathrm{P}$, et al. Socio-economic Aspects of Alzheimer's Disease. Curr Alzheimer Res. 2015;12:903-9011.

6. Scheltens P, et al. Alzheimer's disease. Lancet (London, England). 2016;388:505-17.

7. Alzheimer's Association. 2013 Alzheimer's disease facts and figures. Alzheimers Dement. 2013;9:208-45.

8. Glenner GG, Wong GW. Alzheimer's disease: initial report of the purification and characterization of a novel cerebrovascular amyloid protein. Biochem Biophys Res Commun. 1984;120:885-90.

9. Musiek ES, Holtzman DM. Three dimensions of the amyloid hypothesis: time, space and 'wingmen'. Nat Neurosci. 2015;18:800-6.

10. Ballard C, et al. Alzheimer's disease. Lancet. 2011;377:1019-31.

11. Rafii MS, Aisen PS. Advances in Alzheimer's disease drug development. BMC Med. 2015;13:62.

12. Hung S-Y, Fu W-M. Drug candidates in clinical trials for Alzheimer's disease. J Biomed Sci. 2017;24:47.

13. Cummings J, et al. Alzheimer's disease drug-development pipeline: few candidates, frequent failures. Alzheimers Res Ther. 2014;6:37.

14. Cummings J. Lessons Learned from Alzheimer Disease: Clinical Trials with Negative Outcomes. Clin Transl Sci. 2017:1-6. https://doi.org/10.1111/cts.12491.

15. Wurtman R. Biomarkers in the diagnosis and management of Alzheimer's disease. Metabolism. 2015;64:S47-50. 